\title{
Prediction of left ventricular remodelling by radionuclide imaging
}

\author{
Constantinos D. Anagnostopoulos • Dennis V. Cokkinos
}

Published online: 9 April 2011

(C) Springer-Verlag 2011

Left ventricular remodelling is a complex process by which mechanical, neurohormonal and genetic factors alter ventricular structure and function leading to reduced mechanical performance, electrical instability and sudden death [1]. It is an important aspect of heart failure progression, characterized by dilatation and change of shape of the left ventricle (LV) as well as alterations in the ventricular wall which include hypertrophy, loss of myocytes and increased interstitial fibrosis [2]. At the molecular level, it is characterized by a regression to the fetal pattern, i.e. increase of $\beta$-myosin heavy chain, $\alpha$-actin, atrial natriuretic peptide overexpression, sarco/endoplasmic reticulum $\mathrm{Ca}^{2+}$ ATPase activity decrease and a shift of myocardial metabolism towards glucose utilization [3, 4]. Acute myocardial infarction (MI) is a common cause of LV remodelling. It is estimated that despite primary percutaneous coronary intervention (PCI) and standard current therapy, around $30 \%$ of anterior MIs will develop remodelling. The three major biomechanical mechanisms contributing to the increase of LV volumes over time after MI are: (1) expansion of the infarct in the subacute phase [5], (2) subsequent non-ischaemic infarct extension into the adja-

\footnotetext{
C. D. Anagnostopoulos $(\square)$

Nuclear Medicine Division, PET/CT Department, Clinical

Research Center, Biomedical Research Foundation

Academy of Athens,

4 Soranou Ephessiou St.,

11527 Athens, Greece

e-mail: cdanagnostopoulos@bioacademy.gr

D. V. Cokkinos

Heart and Vessel Cardiovascular Department, Biomedical

Research Foundation Academy of Athens,

4 Soranou Ephessiou St.,

11527 Athens, Greece

e-mail: dcokkinos@bioacademy.gr
}

cent non-infarcted region [6,7] and (3) hypertrophy and dilatation of non-infarcted myocardium in the chronic phase [8-10]. The main factors associated with remodelling are size of infarction, anterior location and late or unsuccessful (or absence of) reperfusion therapy both at the epicardial vessel level and at the microvasculature level. LV remodelling, however, is a potentially reversible or even possibly preventable process. Regression is manifested as a return to a more normal ventricular size and shape and appears to be a good predictor of a reduction in morbidity and mortality $[2,11]$. Several trials on post acute MI patients have demonstrated that this can be achieved by a combination of treatment regimes [12]. This is understandable considering that the three aforementioned mechanisms operate in different regions of the LV and during different time frames after MI, thus making it unlikely for any single drug to be completely effective in addressing all three mechanisms [12]. A major determinant to the selection of the appropriate treatment is the propensity of the underlying MI to result in LV remodelling. Therefore, accurate monitoring of post acute MI patients to identify those who are likely to develop LV remodelling is of great importance.

Based on the mechanistic rationale described above, monitoring is performed with non-invasive imaging and usually includes assessment of heart size, shape and mass, ejection fraction (EF), end-diastolic and end-systolic volumes and regional contractility. Cardiac magnetic resonance (CMR) is the gold standard to assess these parameters as well microvascular obstruction and infarct size. A consistent finding from the CMR literature in the post acute MI setting is that scar size is a key determinant of long-term LV remodelling. Moreover, there are distinct time-dependent patterns of infarct healing and LV remodelling which suggest that the timing of CMR performance is important for assessment of infarct size and prediction of 
LV remodelling [13]. In one study, an infarct size threshold of $24 \%$ has been found to be highly predictive of LV remodelling (sensitivity 92\%, specificity 93\%), and it was also shown that patients with LV remodelling had a greater infarct size reduction, i.e. larger infarct resorption, than patients with preserved LV volumes [14]. Echocardiography is more widely available than CMR and has been used extensively in this setting. Traditionally, prediction of LV remodelling is based on functional parameters derived from echocardiographic studies [15]. Contrast-enhanced echocardiography is more accurate than the unenhanced for predicting LV remodelling, allowing also assessment of infarct size, and more recently, speckle-tracking echocardiography has also been used in the same setting. It was found that impairment of peak LV torsion (a sensitive parameter to measure regional deformation and LV systolic myocardial performance) at $48 \mathrm{~h}$ post acute MI treated with primary PCI had a sensitivity of $95 \%$ and a specificity of $77 \%$ for predicting LV remodelling and complemented the information obtained from infarct size assessment by contrast echocardiography[16]. Single photon emission computed tomography (SPECT) myocardial perfusion imaging (MPI) with ECG gating (ECG-gated SPECT) has been introduced as another option for assessing LV remodelling as it offers the benefit of assessing both perfusion and function in the same myocardial segments as well as scar size, but it also allows accurate and highly reproducible measurements of global LV function and volumes [17]. ECG-gated SPECT is widely available and not expensive and provides technically adequate data in virtually all patients. It has been used previously to assess the effect of primary PCI on LV function and myocardial perfusion. A progressive reduction in infarct size was observed and this was associated with a favourable shortterm evolution of LV function [18]. The relation between infarct severity and LV changes has also been investigated using ECG-gated SPECT, and it was shown that infarct severity was more effective than infarct size for predicting subsequent LV remodelling [19, 20].

Berti et al. in the current issue of the European Journal of Nuclear Medicine and Molecular Imaging [21] have used ECG-gated SPECT (without attenuation correction) to investigate the evolution of perfusion and functional parameters after acute $\mathrm{MI}$ in a group of patients successfully treated with primary PCI. They sought to evaluate (a) the relationship between changes in myocardial perfusion and $\mathrm{LV}$ functional changes at long-term follow-up (average 6 months) and (b) the accuracy of "baseline" perfusion and functional parameters ( 1 month post $\mathrm{MI}$ ) for predicting $\mathrm{LV}$ remodelling at follow-up. They found that patients with remodelling had larger (infarct size $29.3 \pm 7.8 \%$ ) and more transmural (infarct severity $0.28 \pm 0.10$ ) infarctions and reduced LVEF $(35.4 \pm 5.6 \%)$ at baseline, compared to patients without remodelling (infarct size $20.8 \pm 14.4 \%, p<$ 0.05 , infarct severity $0.40 \pm 0.11, p<0.001$, LVEF $44.5 \pm 9.2$, $p<0.001)$. At follow-up, in the group with remodelling, volumes increased significantly $(p<0.001)$, whilst infarct size, infarct severity and LVEF did not change. Conversely, in the group without remodelling, there was a significant decrease in infarct size and transmurality (both $p<0.001$ ) that was accompanied by a significant functional improvement with higher LVEF and smaller volume indexes $(p<0.05$ for all comparisons). In addition, the investigators found that infarct severity, expressing in essence transmurality and residual viability, was the best indicator for predicting $\mathrm{LV}$ remodelling and that the combination of infarct size and severity detected patients with remodelling with $75 \%$ accuracy and 95\% negative predictive value. Infarct resorption (defined as the defect size difference between follow-up and baseline) was comparable between patients with $(-4.4 \pm$ $8.4 \%)$ and without remodelling (6.8 $\pm 9.4 \%)$.

Allowing for the limitations of any such study to unravel fine details of the multifaceted underlying pathophysiology of LV remodelling, the results of the study by Berti et al. show that ECG-gated SPECT can have a role in the assessment of patients 1 month post reperfused acute MI, because it can predict with reasonable accuracy those who are likely to develop LV remodelling. Specific study-related limitations include its retrospective nature and the rather modest number of investigated patients. Despite these drawbacks, such data can be useful to the clinical community and although larger studies are needed before precise guidelines can be formulated, from the findings of the current study and those obtained by other non-invasive imaging modalities, it is clear that accurate quantitative assessment of infarct size and severity and also of LV volumes is valuable for patient monitoring. This can eventually be translated into better care by facilitating the application of personalized medicine, since early identification of LV remodelling could lead to management strategies appropriate for the individual patient's infarct characteristics. To refine these strategies and develop appropriate combinations of treatment regimes or even design novel therapies that can be employed during the optimal time windows necessary to minimize LV remodelling post MI (i.e. specifically targeting infarct expansion as opposed to non-ischaemic infarct extension or compensatory hypertrophy), a multidisciplinary collaboration between physicians, imagers and basic scientists is imperative. Such collaboration can enhance our knowledge on an array of areas relevant to LV remodelling and provide a bidirectional flow of information between bench and bedside. To this end, in-depth investigation of molecular and cellular changes that are translated into structural and functional alterations is of great importance. Equally important are studies on electrical and mechanical determi- 
nants of LV function, particularly in combination with other aspects of cardiac physiology, such as perfusion, metabolism and innervation which can all be assessed by imaging modalities. In this context, nuclear medicine techniques with their ability to track alterations at the molecular or cellular level can play a pivotal role. Their potential can be enhanced even further in the form of hybrid or multimodality imaging that allows different biomarkers to be imaged together, and this is currently being explored both in preclinical and clinical studies.

Beyond the well-validated SPECT or positron emission tomography (PET) techniques used for assessment of perfusion, viability, scar size/severity and function in the post MI setting, there are also other techniques and tracers already in clinical use or approaching the stage of validation in human studies. For example, ${ }^{123}$ I-metaiodobenzylguanidine (MIBG) imaging can be employed to assess neuronal alterations associated with LV remodelling [22], whilst assessment of fatty acid metabolism of the heart and its alterations after MI using $15-\left(p-\left[{ }^{123} \mathrm{I}\right]\right.$ iodophenyl $)$ pentadecanoic acid or its branched-chain analogue, $\beta$ methyl- $p-\left[{ }^{123} \mathrm{I}\right]$ iodophenyl-pentadecanoic acid (BMIPP), is another possibility [23]. At a preclinical level, quantitative radionuclide imaging of matrix metalloproteinase (MMP) activity that is correlated with extracellular matrix degradation is now feasible using radiolabelled molecules targeting MMPs [24]. This can be performed at different time points in combination also with ECG-gated MPI SPECT for monitoring both scar size and MMP activation (both at the MI zone and the remote region) changes over time [25]. Furthermore, such imaging can also be combined with assessment of changes in LV volumes (using radionuclide- or non-radionuclide-based techniques), thus introducing a molecular-mechanical imaging approach that could help to assess in a longitudinal fashion the relationship between enhanced MMP activation after MI and altered regional myocardial deformation[26]. SPECT imaging has also been performed experimentally both with radiolabelled angiotensin-converting enzyme (ACE) inhibitors [27] and angiotensin type 1 antagonists [28]. Early results suggest that changes in the renin-angiotensinaldosterone system that occur after MI can be assessed with these techniques and may be used to predict the risk for developing significant heart failure [27, 28]. In addition, angiogenesis imaging specifically targeting the $\alpha_{\mathrm{v}} \beta_{3}$ integrin to track associated changes in angiogenesis in the setting of LV remodelling (neovessel formation is critical in scar formation whilst the regression of these vessels may be equally important in preventing excessive fibrosis) is another possibility for SPECT imaging [29], as is apoptosis assessment using radiolabelled annexin [30], since apoptotic markers are clearly involved with pathways that can contribute to adverse remodelling after MI [31]. As alluded to earlier, such molecular imaging approaches can provide more useful information in combination with highresolution structural imaging to study with greater precision changes occurring in different myocardial areas. This can be particularly helpful for testing the effect of specific treatment regimes. Accurate evaluation of the complex processes smoldering in the "border" (adjacent noninfarcted) zone is one such example, as this may result in novel tailored, i.e. anti-inflammatory and antiapoptotic therapies, being implemented at an earlier stage [32, 33]. This applies similarly to investigation, at a preclinical level, of parameters other than apoptosis or inflammation (such as MMP activity, angiogenesis, etc....) using the aforementioned imaging techniques to assess treatment response. This can contribute to our understanding of treatment effectiveness at different time points, thus bringing the concept of personalized medicine closer to clinical practice. Such developments are nevertheless by no means imminent, and several steps must take place before the abovementioned novel radiotracers and molecular approaches are applied in the clinical setting or prove their costeffectiveness. Until this happens, monitoring of post acute MI patients will continue to be performed successfully by the more conventional, but nevertheless, state-of-the-art imaging techniques employed by echocardiography, CMR and radionuclide imaging. It is likely that cardiac $\mathrm{CT}$ with its versatility to provide both morphological and functional information as well as potential for tissue characterization will also be utilized in this clinical setting either in a standalone fashion or in a hybrid form [34, 35].

Within the context of the current practice, therefore, the study by Berti et al. [21], despite its limitations, is a step in the right direction. Their results demonstrate that quantification of infarct size and severity using a simple, well-validated and inexpensive technique such as MPI ECG-gated SPECT can predict with reasonable accuracy LV remodelling in acute MI patients treated with primary PCI, thus refining the role of this technique to yet another group of patients in whom its use can influence management decisions.

Conflicts of interest None.

\section{References}

1. Pfeffer MA, Braunwald E. Ventricular remodeling after myocardial infarction. Experimental observations and clinical implications. Circulation 1990;81:1161-72.

2. Sutton MGSJ, Sharpe N. Left ventricular remodeling after myocardial infarction: pathophysiology and therapy. Circulation 2000;101:2981-8.

3. Cokkinos DV, Pantos C. Myocardial remodeling, an overview. Heart Fail Rev 2011;16:1-4. 
4. Javadov S, Rajapurohitam V, Kilić A, Hunter JC, Zeidan A, Said Faruq N, et al. Expression of mitochondrial fusion-fission proteins during post-infarction remodeling: the effect of NHE-1 inhibition. Basic Res Cardiol 2011;106:99-109.

5. Kelley ST, Malekan R, Gorman JH 3rd, Jackson BM, Gorman RC, Suzuki Y, et al. Restraining infarct expansion preserves left ventricular geometry and function after acute anteroapical infarction. Circulation 1999;99(1):135-42.

6. Ratcliffe MB. Non-ischemic infarct extension: a new type of infarct enlargement and a potential therapeutic target. J Am Coll Cardiol 2002;40:1168-71.

7. Jackson BM, Gorman JH, Moainie SL, Guy TS, Narula N, Narula $\mathrm{J}$, et al. Extension of borderzone myocardium in postinfarction dilated cardiomyopathy. J Am Coll Cardiol 2002;40(6):1160-7.

8. Cheng A, Nguyen TC, Malinowski M, Langer F, Liang D, Daughters GT, et al. Passive ventricular constraint prevents transmural shear strain progression in left ventricle remodeling. Circulation 2006;114(1 Suppl):I79-86.

9. Kramer CM, Rogers WJ, Park CS, Seibel PS, Shaffer A, Theobald $\mathrm{TM}$, et al. Regional myocyte hypertrophy parallels regional myocardial dysfunction during post-infarct remodeling. J Mol Cell Cardiol 1998;30(9):1773-8.

10. Saeed M, Lee RJ, Weber O, Do L, Martin A, Ursell P, et al. Scarred myocardium imposes additional burden on remote viable myocardium despite a reduction in the extent of area with late contrast MR enhancement. Eur Radiol 2006;16(4):827-36.

11. Cohn JN, Ferrari R, Sharpe N. Cardiac remodeling — concepts and clinical implications: a consensus paper from an international forum on cardiac remodelling. Behalf of an International Forum on Cardiac Remodeling. J Am Coll Cardiol 2000;35(3):569-82.

12. French BA, Kramer CM. Mechanisms of post-infarct left ventricular remodeling. Drug Discov Today Dis Mech 2007;4 (3):185-96

13. Ørn S, Manhenke C, Greve OJ, Larsen AI, Bonarjee VV, Edvardsen $\mathrm{T}$, et al. Microvascular obstruction is a major determinant of infarct healing and subsequent left ventricular remodelling following primary percutaneous coronary intervention. Eur Heart J 2009;30(16):1978-85.

14. Lund GK, Stork A, Muellerleile K, Barmeyer AA, Bansmann MP, Knefel M, et al. Prediction of left ventricular remodeling and analysis of infarct resorption in patients with reperfused myocardial infarcts by using contrast-enhanced MR imaging. Radiology 2007;245:95-102.

15. Bolognese L, Neskovic AN, Parodi G, Cerisano G, Buonamici P, Santoro GM, et al. Left ventricular remodeling after primary coronary angioplasty: patterns of left ventricular dilation and longterm prognostic implications. Circulation 2002;106:2351-7.

16. Nucifora G, Marsan NA, Bertini M, Delgado V, Siebelink HM, van Werkhoven JM, et al. Reduced left ventricular torsion early after myocardial infarction is related to left ventricular remodeling. Circ Cardiovasc Imaging 2010;3(4):433-42.

17. Zellweger MJ, Tabacek G, Zutter AW, Weinbacher M, Cron TA, Müller-Brand J, et al. Evidence for left ventricular remodeling after percutaneous coronary intervention: effect of percutaneous coronary intervention on left ventricular ejection fraction and volumes. Int J Cardiol 2004;96(2):197-201.

18. Castro PF, Corbalan R, Baeza R, Nazzal C, Greig DP, Miranda FP, et al. Effect of primary coronary angioplasty on left ventricular function and myocardial perfusion as determined by Tc- $99 \mathrm{~m}$ sestamibi scintigraphy. Am J Cardiol 2001;87:1181-4.

19. Lipiecki J, Cachin F, Durel N, de Tauriac O, Ponsonnaille J, Maublant J. Influence of infarct-zone viability detected by rest Tc$99 \mathrm{~m}$ sestamibi gated SPECT on left ventricular remodeling after acute myocardial infarction treated by percutaneous transluminal coronary angioplasty in the acute phase. J Nucl Cardiol 2004;11:673-81.
20. Ernande L, Cachin F, Chabrot P, Durel N, Morand D, Boyer L, et al. Rest and low-dose dobutamine Tc-99m-mibi gated-SPECT for early prediction of left ventricular remodeling after a first reperfused myocardial infarction. J Nucl Cardiol 2009;16:597604.

21. Berti V, Sciagrà R, Acampa W, Ricci F, Cerisano G, Gallicchio R, et al. Relationship between infarct size and severity measured by gated SPECT and long-term left ventricular remodelling after acute myocardial infarction. Eur $\mathrm{J}$ Nucl Med Mol Imaging. doi:10.1007/s00259-011-1739-7.

22. Ohte N, Kurokawa K, Iida A, Narita H, Akita S, Yajima K, et al. Myocardial oxidative metabolism in remote normal regions in the left ventricles with remodeling after myocardial infarction: effects of beta-adrenoceptor blockers. J Nucl Med 2002;43(6):780-5.

23. Hashimoto A, Nakata T, Tamaki N, Kobayashi T, Matsuki T, Shogase T, et al. Serial alterations and prognostic implications of myocardial perfusion and fatty acid metabolism in patients with acute myocardial infarction. Circ J 2006;70(11):1466-74.

24. Su H, Spinale FG, Dobrucki LW, Song J, Hua J, Sweterlitsch S, et al. Noninvasive targeted imaging of matrix metalloproteinase activation in a murine model of postinfarction remodeling. Circulation 2005;112:3157-67.

25. Liu Y, Sahul Z, Weyman C, Ryder WJ, Dione DP, Dobrucki WL, et al. Hotspot quantification of myocardial focal tracer uptake from molecular targeted SPECT/CT images: canine validation. Proc SPIE 2008;6915:69150N1-8.

26. Kramer CM, Sinusas AJ, Sosnovik DE, French BA, Bengel FM. Multimodality imaging of myocardial injury and remodeling. $\mathrm{J}$ Nucl Med 2010;51:107S-21S.

27. Dilsizian V, Eckelman WC, Loredo ML, Jagoda EM, Shirani J. Evidence for tissue angiotensin-converting enzyme in explanted hearts of ischemic cardiomyopathy using targeted radiotracer technique. J Nucl Med 2007;48:182-7.

28. Verjans JW, Lovhaug D, Narula N, Petrov AD, Indrevoll B, Bjurgert E, et al. Noninvasive imaging of angiotensin receptors after myocardial infarction. JACC Cardiovasc Imaging 2008;1:354-62.

29. Higuchi T, Bengel FM, Seidl S, Watzlowik P, Kessler H, Hegenloh R, et al. Assessment of alphavbeta3 integrin expression after myocardial infarction by positron emission tomography. Cardiovasc Res 2008;78(2):395-403.

30. Doue T, Ohtsuki K, Ogawa K, Ueda M, Azuma A, Saji H, et al. Cardioprotective effects of erythropoietin in rats subjected to ischemia-reperfusion injury: assessment of infarct size with 99mTc-annexin V. J Nucl Med 2008;49:1694-700.

31. Ekhterae D, Hinmon R, Matsuzaki K, Noma M, Zhu W, Xiao RP, et al. Infarction induced myocardial apoptosis and ARC activation. J Surg Res 2011;166(1):59-67.

32. Gilson WD, Epstein FH, Yang Z, Xu Y, Prasad K-MR, Toufektsian $\mathrm{M}-\mathrm{C}$, et al. Borderzone contractile dysfunction is transiently attenuated and left ventricular structural remodeling is markedly reduced following reperfused myocardial infarction in inducible nitro oxide synthase knockout mice. J Am Coll Cardiol 2007;50:1799-807.

33. Biondi-Zoccai GGL, Abbate A, Bussani R, Camilot D, Giorgio FD, Marino M-PD, et al. Reduced post-infarction myocardial apoptosis in women: a clue to their different clinical course? Heart 2005;91:99-101.

34. Rodriguez-Granillo GA, Rosales MA, Baum S, Rennes P, Rodriguez-Pagani C, Curotto V, et al. Early assessment of myocardial viability by the use of delayed enhancement computed tomography after primary percutaneous coronary intervention. JACC Cardiovasc Imaging 2009;2:1072-81.

35. Flachskampf FA, Schmid M, Rost C, Achenbach S, DeMaria AN, Daniel WG. Cardiac imaging after myocardial infarction. Eur Heart J 2011;32:272-83. 